\title{
Evaluation of Expression Patterns of Cytokeratin and Mucin Peptide Core Antigen for Diagnostic Role in Upper Gastrointestinal Tract Pre Neoplastic and Neoplastic Lesions
}

\author{
Mallika Dixit $^{1}$, Shruti Sharma ${ }^{1}$, Zeeba Shamim Jairajpuri ${ }^{2 *}$, Sujala Kapur ${ }^{1}$, Usha Agrawal ${ }^{1}$, \\ Neelima Jain ${ }^{3}$, Manisha Thakur ${ }^{3}$, B.K Tripathi ${ }^{3}$ \\ ${ }^{1}$ National Institute of Pathology,Indian Council of MedicalResearch, Safdarjung Hospital Campus,New Delhi 110029. \\ ${ }^{2}$ Department of Pathology Hamdard Institute of Medical Sciences and Research Jamia Hamdard, New Delhi, India \\ ${ }^{3}$ Department of Medicine VMMC and Safdarjung Hospital, New Delhi India
}

\begin{abstract}
Introduction: Gastrointestinal tract (GIT) lesions include a wide variety of lesions, which may be diagnostically challenging on histopathology and may be difficult to differentiate without the aid of immunohistochemical stains.

Objective: The present study was undertaken to histopathologically analyze the upper GIT lesions and determine the expression pattern of cytokeratin (CK) and mucin peptide core antigen (MUC) in these lesions and their usefulness in diagnosis.

Material and Methods: A 135 cases with clinical diagnosis of upper GIT lesions including Barrett's metaplasia (BM), carcinoma esophagus, gastric intestinal metaplasia (GIM) and gastric carcinoma were included. Standard diagnostic criteria were used in evaluating tissue sections and arriving at a diagnosis. Relevant clinical data including age, gender, complications and symptoms of disease were noted. Immunohistochemical evaluation of the lesions were done. The antibody panel included CK7,CK18, CK19, CK20, MUC1\&MUC2.

Results: Immunohistochemical pattern CK7-/CK20+ was seen in 85\% gastric adenocarcinoma and 82\% gastric intestinal metaplasia. CK7+/ CK20- was characteristic of esophageal adenocarcinoma. CK7+/CK20+ pattern was characteristic of Barrett's metaplasia. MUC1 and MUC2 expression was seen in both goblet and non goblet cells in Barrett's metaplasia, MUC1 in both gastric intestinal metaplasia and MUC2 in only goblet cells. MUC2 is a good marker of mucinous carcinomas of esophagus and stomach.

Conclusions: Pre-neoplastic and neoplastic lesions of the gastrointestinal tract are varied yet histologically challenging, often prove to be a diagnostic dilemma. Understanding unique immunohistochemical profiles of each, combined with histopathological and endoscopic correlation greatly assists in the diagnosis and management of these lesions.
\end{abstract}

Keywords: Immunohistochemical, Cytokeratin, Muc, Gastrointestinal Lesions

\section{Introduction}

Upper gastrointestinal tract is an important site for wide variety of lesions forming some of the most commonly encountered problems in clinical practice. To facilitate diagnosis of different gastric lesions, endoscopy and histology complement each other, hence playing an important role in the diagnosis of upper GIT neoplasms and inflammatory lesions. This aids in early management and monitoring the course and extent of the disease, response of therapy and early detection of complications. ${ }^{[1]}$

GIT lesions also include a wide variety of vastly different tumors which often present in late stages as distant metastases. These lesions may be diagnostically challenging on histopathology, it may be difficult to differentiate them without the aid of immunohistochemical stains. ${ }^{[2]}$ since most of them have unique immunohistochemical profiles. The role of immunohistochemical stains is an important one, especially in determining the origin and differentiation of GIT lesions. ${ }^{[2]} \mathrm{CKs}$ are the most fundamental markers of epithelial differentiation, classification of tumors by their specific types of intermediate filament $\mathrm{CK}$ has recently become very valuable in clinical histo-diagnosis. ${ }^{[3]}$ Expression of CK of normal epithelial cells of the upper GIT changes due to various kinds of damage, which may sometimes not be morphologically evident hence aiding in diagnosis.

Mucins on the other hand have an important role in the defense of mucosa of the upper GIT against the insult of acid, pepsin and bile. Changes in the expression of mucins occur in patients with various GIT lesions. ${ }^{[4]}$ MUCs have differential expression patterns which are tissue specific in the GIT, these patterns can be used to classify different phenotypes of adenocarcinoma. MUC expression patterns are also known to carry prognostic significance and is 
known to be deregulated in lesions especially Barrett's metaplasia. ${ }^{[5]}$ In small endoscopic biopsies, distinguishing lesions may be difficult as a result of scant tissue and biopsy artifact.

The objective of the present work was to histopathologically analyze the upper GIT lesions and also determine the expression pattern of cytokeratin and mucin peptide core antigen in these lesions to assist in diagnosis.

\section{Material and Methods}

This cross sectional study was conducted in a tertiary care hospital and its affiliated institute, on biopsies and resected specimens received from patients with symptoms of upper GIT lesions presenting at the Department of Medicine and Surgery over a period of three years. Patients with clinical diagnosis of upper GIT lesions like BM, carcinoma esophagus, gastric intestinal metaplasia and gastric carcinoma were recruited.

A total of 135 cases were included in the study. Lesions which did not fit into the diagnostic criteria of the above diseases were excluded from the study. Formalin fixed samples were processed and embedded for preparing paraffin sections. Serial tissue sections were stained for histopathological diagnosis. The sections were stained with haematoxylin and eosin (H\&E) as well as Periodic acid Schiff (PAS), Alcian blue $\mathrm{pH} 2.5$ and Alcian blue $\mathrm{pH}$ 0.5 to identify neutral mucin, sialomucin and sulphomucin respectively. GIM as well as BM are established premalignant conditions, characterized by mucin carbohydrate modifications defined by histochemical methods. Standard diagnostic criteria were used in evaluating tissue sections and arriving at a diagnosis. Biopsies from normal areas served as control. Clinical data including age, gender, complications and symptoms of disease were also noted.

Immunohistochemical evaluation of the tissue was done, sections were obtained on poly-L-lysine coated slides and were subjected to immunohistochemical staining using mono/polyclonal antibodies by $\mathrm{ABC}$ method using diaminobenzidine (DAB) as substrate. The antibody panel included CK7,CK18,CK19,CK20, MUC1\&MUC2. The interpretation of immunohistochemical staining was done by two observers. Cases exhibiting more than 5\% positivity for the antibody were taken as positive. Relevant statistical analysis was done Chi square and Fisher exact test was applied as tests of significance, a p-value of $<0.05$ was considered as significant value.

\section{Results}

A total of 135 endoscopic biopsies and surgical resected specimens were received in the histopathology section. Out of these 135 samples, 78 were from esophagus ( 66 biopsies and 12 resected specimens) and 57 cases from stomach (47 biopsies and 10 resected specimens).

The mean age was similar in all age groups (Barrett's oesophagus:57 years, Intestinal metaplasia: 56 years, Squamous cell carcinoma oesophagus 55.3 years, Adenocarcinoma oesophagus:52 years) except that of gastric adenocarcinoma where it was 45 years, however, the age range of the patients was seen to vary from the $2^{\text {nd }}$ to the $8^{\text {th }}$ decade. The peak incidence was seen to be $>50$ years in all the lesions. (Table-1) A male predominance was observed in both esophageal and gastric pathologies.

Metaplastic lesions evaluated included gastric intestinal metaplasia (GIM) and Barrett's metaplasia (BM) both are established pre-malignant conditions, characterized by mucin carbohydrate modifications defined by histochemical methods. Based on histochemical staining the both lesions were classified into complete (Type I) and incomplete type (Type II,III) of intestinal metaplasia. (Table 2) Complete intestinal metaplasia (CIM) was found to be significantly higher in GIM as compared to BM, $\mathrm{p}=0.01$ whereas incomplete intestinal metaplasia (IIM) was significantly higher in $\mathrm{BM}(\mathrm{p}=0.01)$.

Immunohistochemical evaluation for expression pattern of CK and MUC was done in both BM and GIM. (Figures-1,2,3) 20 cases of normal esophageal epithelium and 18 cases of normal gastric mucosa were studied for normal expression of CK7, CK18, CK19, CK20, MUC1 \& MUC2. A significant correlation $(\mathrm{p}<0.05)$ was seen on comparing expression of CK7, CK20, CK18 in normal gastric and esophageal mucosa. Moreover, CK7, CK20 and MUC2 were found to be significantly expressed in BM $(p<0.05)$ when compared to normal esophageal mucosa. On comparing expression of immunohistochemical markers of normal gastric mucosa to GIM a significant decrease in CK7 and MUC1 expression $(\mathrm{p}<0.05)$ was noted while an increased expression of CK20 and MUC2 $(\mathrm{p}<0.05)$ in GIM as compared to normal was seen. (Table 3 )

Barrett's metaplasia showed CK7 immunostaining in the superficial and deep glands in $94 \%$ of the cases while CK20 was seen only in the surface epithelium in $66.7 \%$ cases. This pattern of staining is characteristic of BM. 33\% of the cases were negative for CK20, none of the cases showed CK20 immunoreactivity of both superficial and deep glands and surface epithelium a pattern characteristic of GIM. 5.6\% cases were negative for both markers. Strong diffuse CK7 staining and superficial CK20 immunostaining was seen in IIM of BM. Expression of MUC in BM was analyzed, MUC1 was not expressed while MUC2 was detected in all the cases in goblet and columnar cells in CIM. In IIM of BM MUC1 was seen in $93 \%$ cases in both 
goblet and columnar cells while MUC 2 was expressed in all the cases. Gastric Intestinal metaplasia, on the other hand showed CK20 immunoreactivity (94\%) of both superficial and deep glands along with surface epithelium. Lack of CK7 staining was seen in majority of the GIM (88\%). Only $11.8 \%$ cases showed patchy CK7 staining and diffuse CK20 in superficial and deep glands. None of the cases showed characteristic BM pattern. On analyzing the staining of intestinal metaplasia of GIM, CIM showed strong diffuse CK20 staining while there was lack of CK7 expression, IIM on the other hand showed weak patchy CK7 staining and moderate immunostaining with CK20. Immunodetection of MUC in Type I metaplasia (CIM) of GIM revealed no expression of MUC1 in goblet and columnar cells while MUC 2 was seen in all the cases in goblet cells. MUC1 expression was seen in all of the IIM cases of GIM analyzed in both goblet and columnar cells while MUC2 was seen in only goblet cells of all the cases. (Figure 4)
Immunohistochemical marker profile was also evaluated in gastric and esophageal carcinoma. (Table-4, Figures 5,6,7) In gastric adenocarcinomas, expression patterns of $\mathrm{CK}$ and MUC were evaluated (Fig-9). CK7 expression was seen to be significantly decreased in gastric adenocarcinoma compared to normal mucosa, while CK20 was increased, however CK18 showed no significant difference on comparison. CK 19 also showed a decreased expression, MUC2 however was also significantly expressed.

Esophageal cancers on the other hand showed increasing CK7 and CK18 expression while CK20 had restricted expression in cancers as compared to normal mucosa where its not expressed at all. MUC2 expression was however significantly increased in esophageal adenocarcinoma. MUC1expresssion was seen to be decreased in esophageal SCC. (Fig-10,11) CK7, CK18, CK19, CK20, MUC1 \& MUC2 exhibited cytoplasmic and membranous positivity. In each case $>5 \%$ cells showing positivity for the marker was taken as positive.

Table1:Distribution Of Cases According To Diagnosis, Sex Ratio \& Age Incidence.

\begin{tabular}{|c|c|c|c|c|c|c|}
\hline \multirow{2}{*}{ Diagnosis } & No. of Cases & Male: & Age Range in yrs & \multicolumn{3}{|c|}{ Age Groups in Yrs (\%) } \\
\cline { 5 - 7 } & (N= 135) & Female & (Mean Age) & $<30$ & $30-50$ & \multicolumn{3}{|c|}{$\mathbf{5 0}$} \\
\hline Barrett's Metaplasia & 18 & $8: 1$ & $28-84(57)$ & $1(5.6)$ & $3(16.7)$ & $14(77.8)$ \\
\hline Adenocarcinoma Esophagus & 20 & $4: 1$ & $22-80(52)$ & $2(10)$ & $4(20)$ & $14(70)$ \\
\hline SCC $^{*}$ Esophagus & 40 & $2.3: 1$ & $22-82(55.3)$ & $1(2.5)$ & $10(25)$ & $29(72.5)$ \\
\hline Gastric Intestinal Metaplasia & 17 & $4.7: 1$ & $29-83(56)$ & $1(5.9)$ & $3(17.7)$ & $13(76.4)$ \\
\hline Gastric Adenocarcinoma & 40 & $3: 1$ & $21-71(45)$ & $2(5)$ & $15(37.5)$ & $23(57.5)$ \\
\hline
\end{tabular}

*SCC: Squamous Cell Carcinoma

Table 2: Distribution of cases according to type metaplasia in esophagus and stomach

\begin{tabular}{|c|c|c|c|c|}
\hline \multirow{2}{*}{ Lesion } & \multirow{2}{*}{ No. of Cases } & $\begin{array}{c}\text { Complete Intestinal } \\
\text { Metaplasia- Type I (\%) }\end{array}$ & \multicolumn{3}{|c|}{ Incomplete Intestinal Metaplasia (\%) } \\
\cline { 3 - 5 } & & $2(11)$ & $14(77.8)$ & Type II \\
\hline Barrett's esophagus & 18 & $14(82.3)$ & $2(11.8)$ & $1(11.2)$ \\
\hline Gastric Intestinal Metaplasia & 17 & $\mathbf{1 6 ( 4 5 . 7 )}$ & $\mathbf{1 6 ( 4 5 . 7 )}$ & $\mathbf{3 ( 8 . 6 )}$ \\
\hline Total & $\mathbf{3 5}$ & &
\end{tabular}

Table 3: Expression Pattern Of Immunohistochemical Markers In Normal Mucosa \& Metaplastic Lesions.

\begin{tabular}{|c|c|c|c|c|c|c|}
\hline Antibody & $\begin{array}{l}\text { Normal Esophageal } \\
\text { Mucosa, } n=20 .(\%)\end{array}$ & $\begin{array}{c}\text { Barrett's } \\
\text { Metaplasia, } n=18(\%)\end{array}$ & $P$ value & $\begin{array}{c}\text { Normal Gastric } \\
\text { Mucosa, } n=18 .(\%)\end{array}$ & $\begin{array}{c}\text { Gastric Intestinal } \\
\text { Metaplasia, } n=17 .(\%)\end{array}$ & $P$ value \\
\hline CK7 & $1(5)$ & $17(94)$ & $<0.05$ & $14(77)$ & $2(11.7)$ & $<0.05$ \\
\hline CK20 & 0 & $12(66.7)$ & $<0.05$ & $7(38.9)$ & 16(94) & $<0.05$ \\
\hline CK18 & $3(15)$ & $8(44)$ & ${ }^{*} \mathrm{NS}$ & $17(94)$ & $14(77.8)$ & NS \\
\hline CK19 & $17(85)$ & $13(72.2)$ & NS & $16(88.9)$ & $12(70.6)$ & NS \\
\hline MUC1 & $17(85)$ & $15(83)$ & NS & $18(100)$ & $4(23.5)$ & $<0.05$ \\
\hline MUC2 & 0 & $18(100)$ & $<0.05$ & 0 & $17(100)$ & $<0.05$ \\
\hline
\end{tabular}

*NS: Not Significant 
Table-4 : Immunohistochemical Marker profile in Gastric and Esophageal Carcinoma.

\begin{tabular}{|c|c|c|c|c|c|}
\hline Antibody & $\begin{array}{c}\text { Normal Gastric } \\
\text { Mucosa, } \mathbf{n = 1 8 .} \\
(\%)\end{array}$ & $\begin{array}{c}\text { Gastric } \\
\text { Adenocarcinoma } \\
\mathbf{n = 4 0}(\mathbf{\%})\end{array}$ & $\begin{array}{c}\text { Normal } \\
\text { Esophageal } \\
\text { Mucosa, } \mathbf{n = 2 0 . ( \% )}\end{array}$ & $\begin{array}{c}\text { Esophageal } \\
\text { Adenocarcinoma } \\
\mathbf{n = 2 0}(\%)\end{array}$ & $\begin{array}{c}\text { Esophageal SCC } \\
\mathbf{n = 4 0}(\%)\end{array}$ \\
\hline CK7 & $14(77)$ & $4(10)$ & $1(5)$ & $17(85)$ & $12(30)$ \\
\hline CK20 & $7(38.9)$ & $38(95)$ & $0(0)$ & $3(15)$ & $2(5)$ \\
\hline CK18 & $17(94)$ & $38(95)$ & $3(15)$ & $18(90)$ & $38(95)$ \\
\hline CK19 & $16(88.9)$ & $16(40)$ & $17(85)$ & $16(80)$ & $33(82)$ \\
\hline MUC1 & $18(100)$ & $33(82)$ & $17(85)$ & $17(85)$ & $20(50)$ \\
\hline MUC2 & $0(0)$ & $34(85)$ & $0(0)$ & $8(40)$ & $1(2.54)$ \\
\hline
\end{tabular}

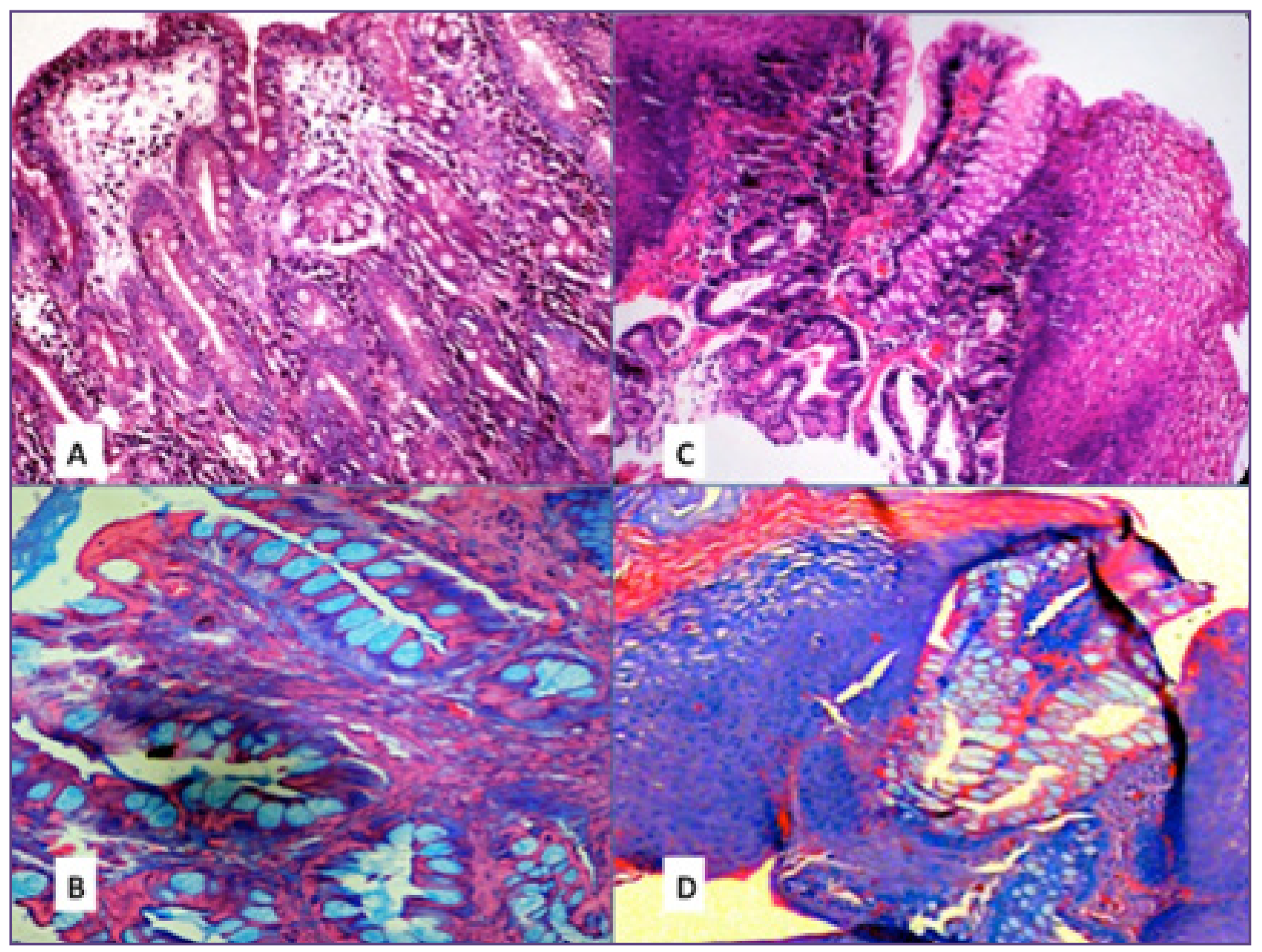

Fig 1:A: Gastric intestinal metaplasia H\&E (20X) B: Complete GIM alcian blue positive pH2.5 (40X) C: Barrett's Metaplasia H\&E (10X) D: AB PAS showing alcian blue positive goblet cell in incomplete metaplasia (20 X). 


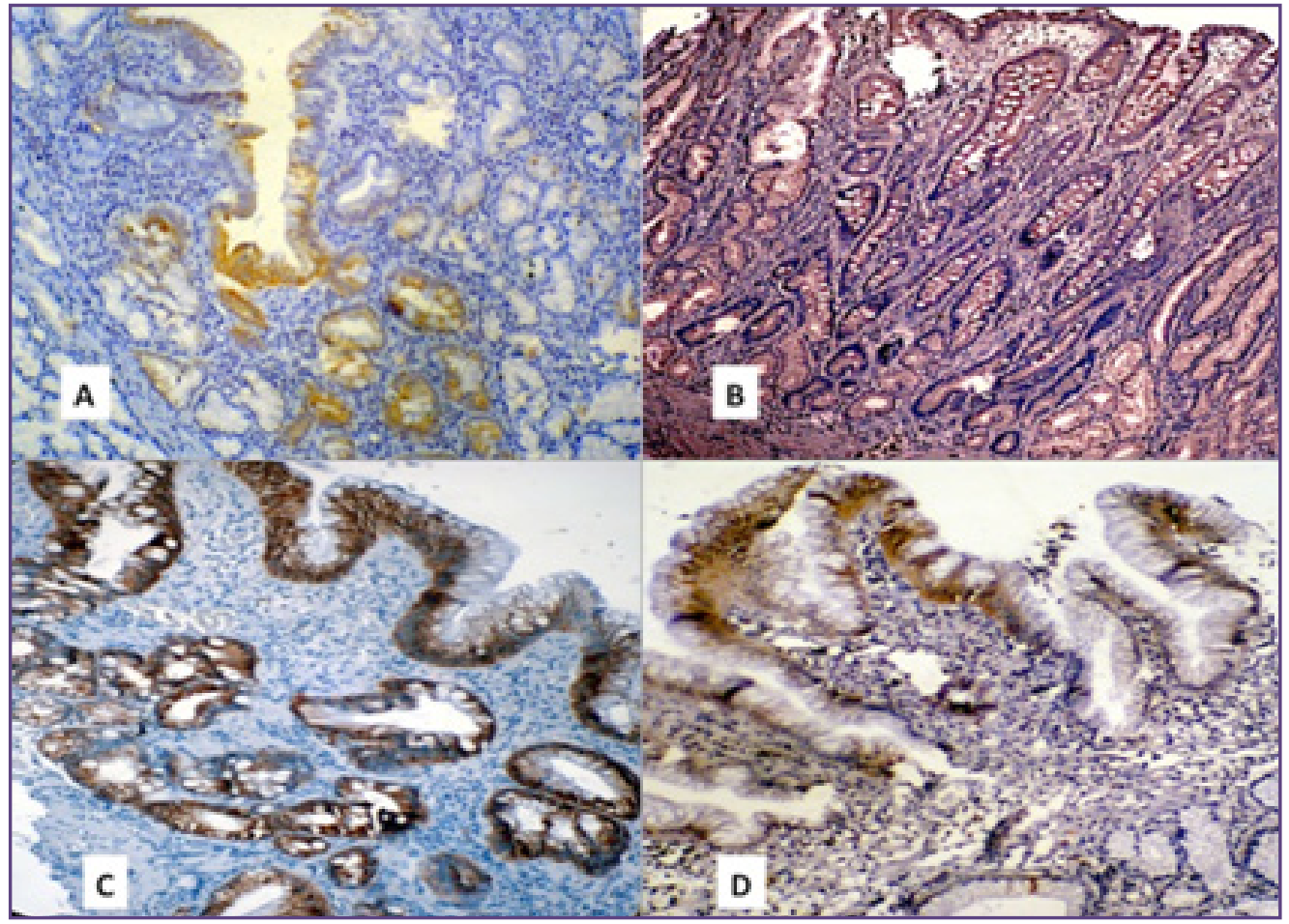

Fig. 2:A: Patchy cytoplasmic CK7 positivity in GIM (20X)B: Diffuse cytoplasmic CK20 positivity in GIM (10X)C:Strong and diffuse cytoplasmic CK7 positivity in BM (20X)D: Superficial CK20 positivity in BM (40X).

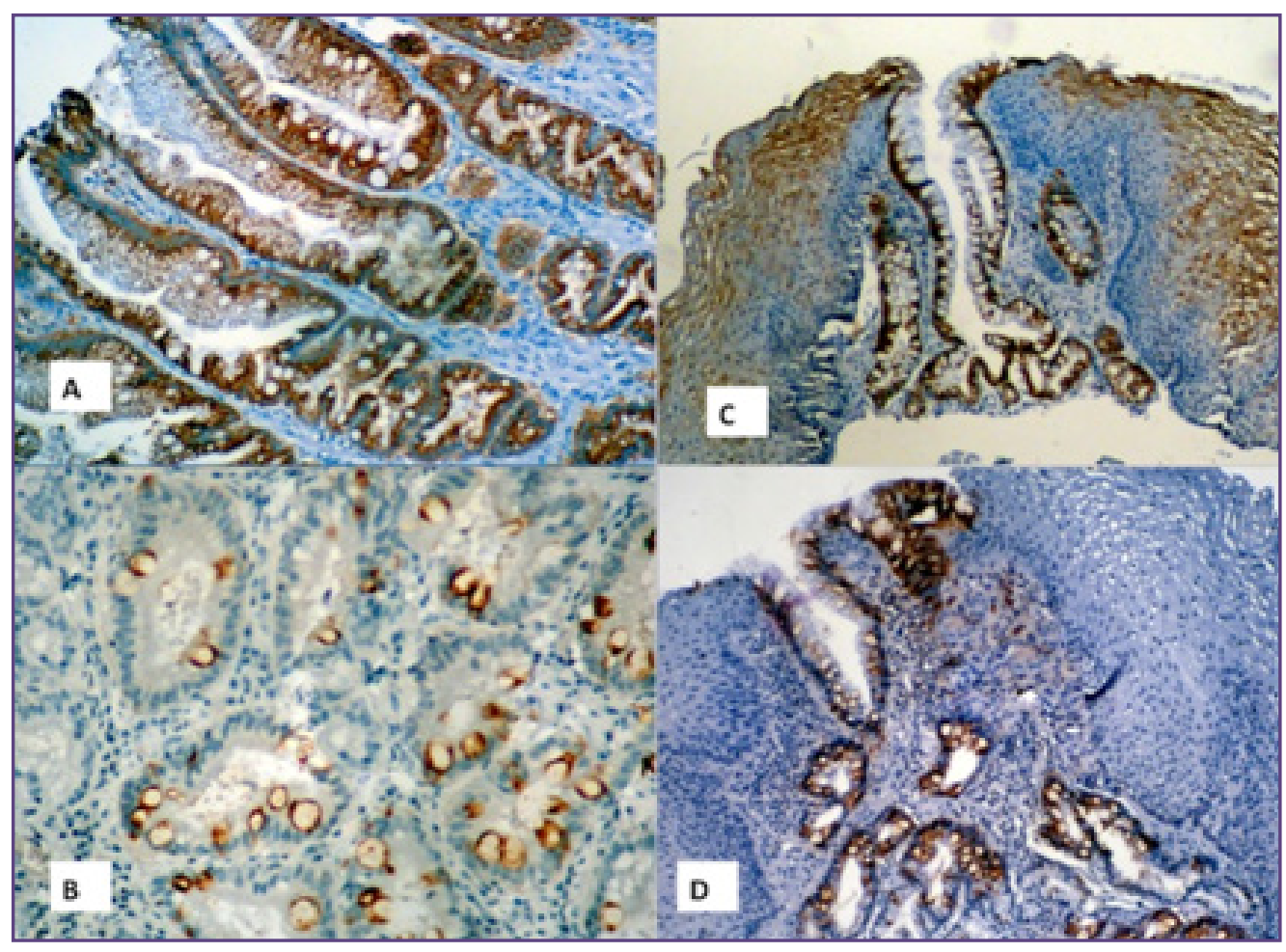

Fig. 3:A:Diffuse and strong cytoplasmic MUC1 positivity in GIM, IIM (20X)B: Membranous MUC2 positivity in goblet cells only in GIM (40X)C: Strong \& diffuse cytoplasmic \& membranous MUC1 positivity in BM \& in normal esophageal mucosa (10 X)D:Strong \& diffuse cytoplasmic \& membranous MUC2 positivity in BM absent in normal esophageal mucosa (10 X) 


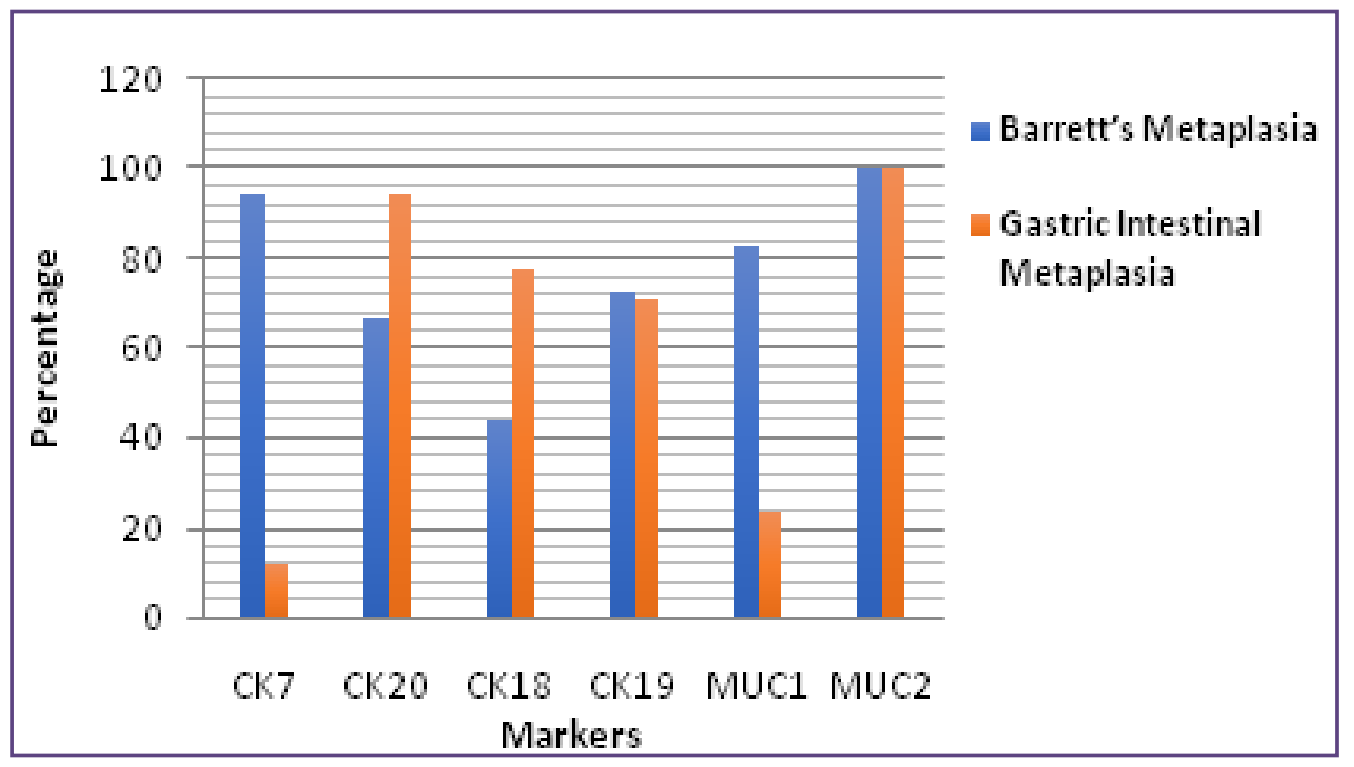

Fig. 4: Graph representing Immunohistochemical Marker profile in Barrett's and Gastric Intestinal Metaplasia.

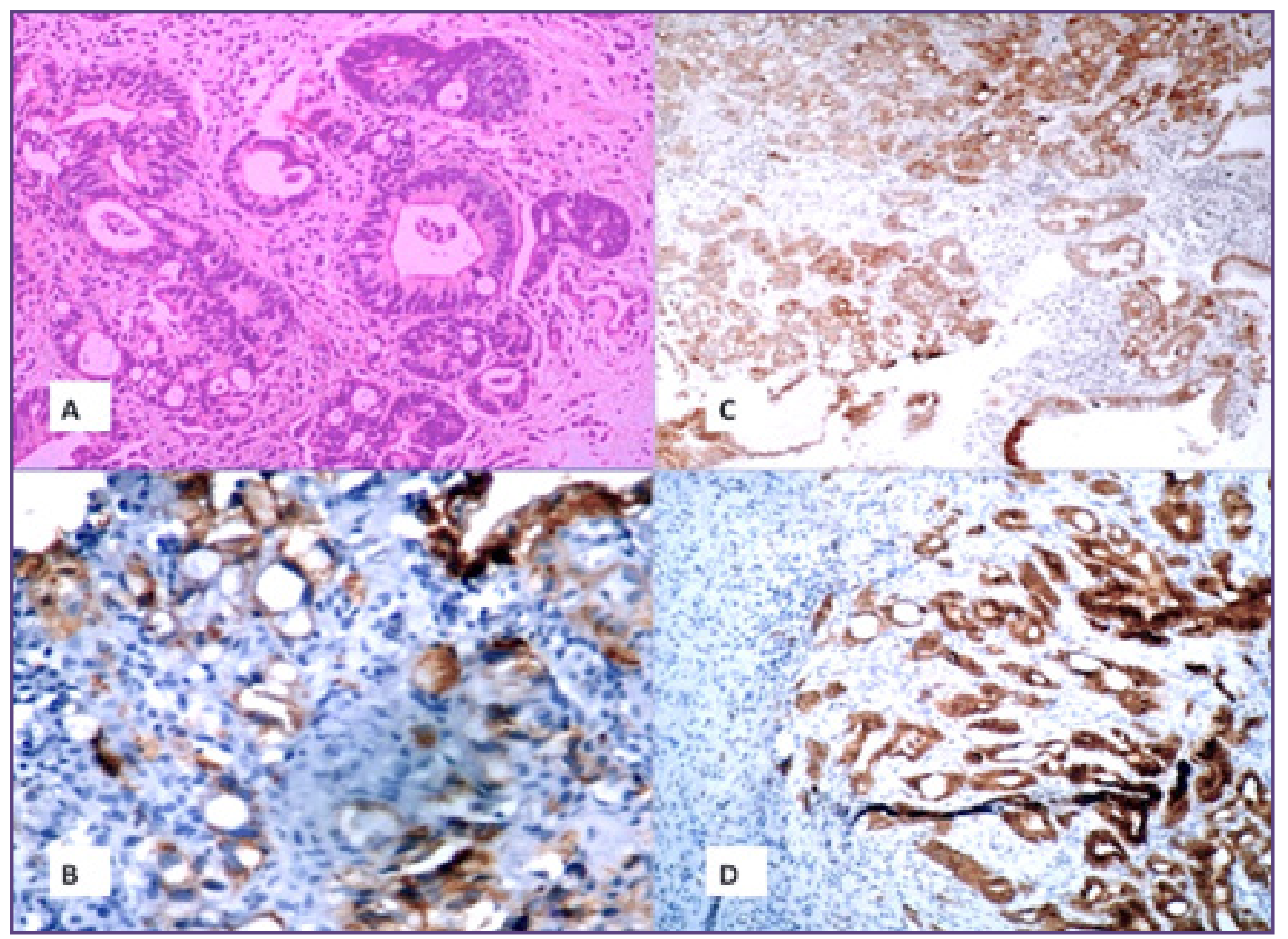

Fig. 5:A: Well differentiated gastric adenocarcinoma H\&E (40X)B: CK20 expression in Well Differentiated Adenocarcinoma, CK7 was negative.C: MUC1expression in Gastric AdenocarcinomaD: MUC2expression in Gastric Adenocarcinoma. 


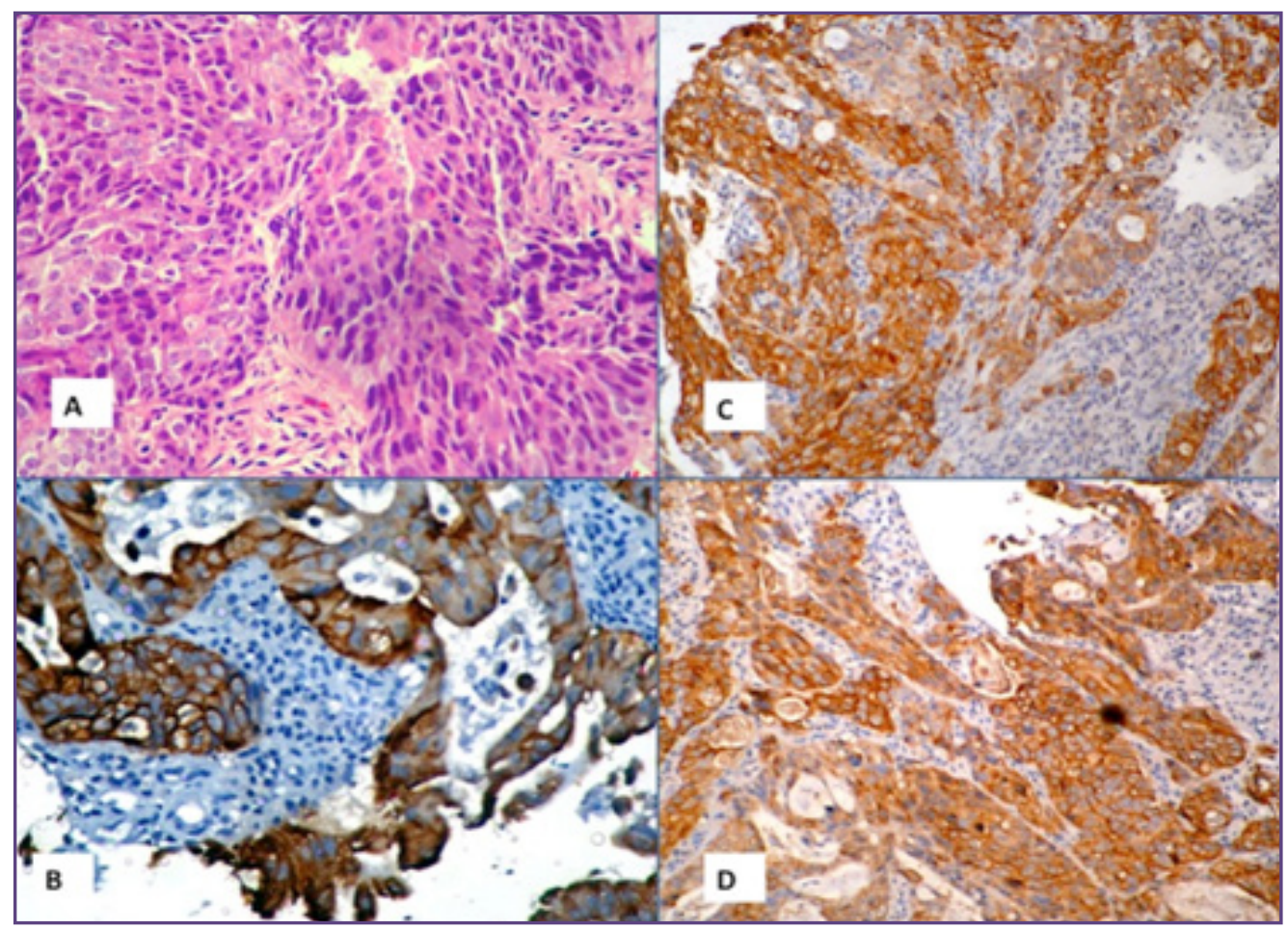

Fig. 6: A:Moderately differentiated squamous cell carcinoma H\&E (40X) B: Cytoplasmic \& membranous CK 19 positivity (40X) C: Cytoplasmic CK18 positivity (10X)D:Cytoplasmic \& membranous MUC1 positivity (20X).

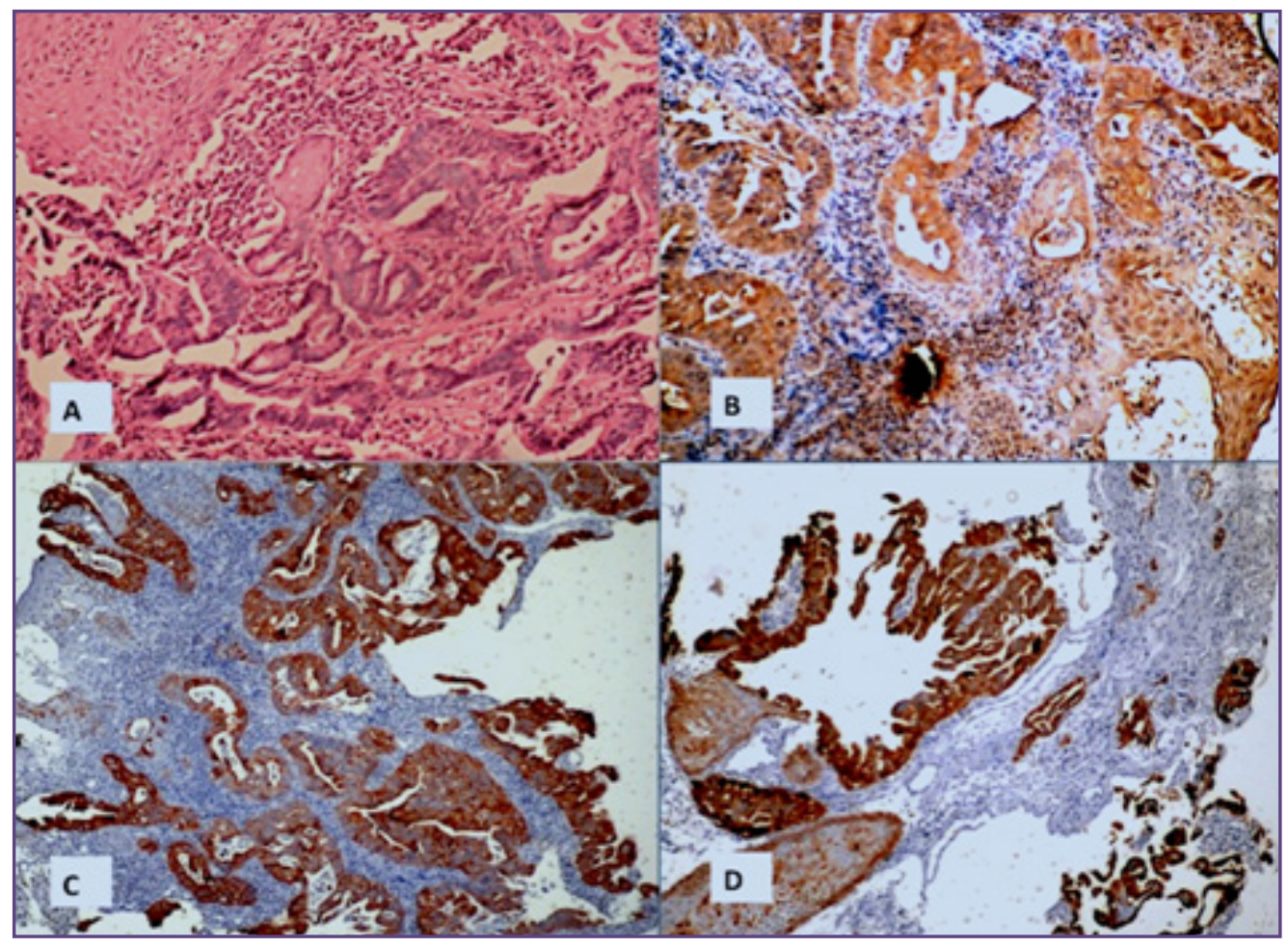

Fig. 7A: Esophageal adenocarcinoma H\&E (20X)B:Cytoplasmic MUC1 positivity (10X) C: Cytoplasmic CK18 positivity (40X) D:Cytoplasmic CK19 positivity (20X). 


\section{Discussion}

Upper GIT lesions are commonly encountered in clinical practice, endoscopic findings and biopsy play an important role in diagnosis and subsequent management of the diseases. Amongst the many lesions in this region, our study was limited to Barrett's metaplasia/esophagus, gastro-intestinal metaplasia and malignancies of the esophagus and stomach. Lesions analyzed in the present study, showed a male predominance with majority of the patients $>50$ years of age, (Table I) this was in concurrence with other authors. ${ }^{[6,7]}$ where the authors observed a mean age of $>50$ years and a male predilection. Similarly in a study, the majority of the patients were male and were less than 65 years of age. ${ }^{[8]}$

Whether histology of the squamo-columnar junction and that of the cardiac mucosa represent normal anatomical segment or are an acquired condition is still disputed. ${ }^{\left[{ }^{9}\right]} \mathrm{BE}$ and GIM of the cardiac mucosa cannot be differentiated histologically as both lesions are characterized by the presence of intestinal metaplasia (IM) with goblet cells. Endoscopy plays a crucial role in differentiating the two lesions, $\mathrm{BE}$ is diagnosed by visible tongues of mucosa of any length, while absence of same as GIM..$^{[9,10]}$ Short segment $\mathrm{BE}(<3 \mathrm{~cm}$ in length) and ultrashort segment $\mathrm{BE}$ are more difficult to recognize by endoscopy, potentially being confused with GIM. ${ }^{[1]}$ The significance of establishing a diagnosis of Barrett's esophagus and intestinal metaplasia lies in the fact that these conditions carry with them a risk of progression to malignancy. Diagnostic challenges include improper sampling, reactive changes and interobserver variation in interpretation of dysplastic changes. In order to arrive at the correct diagnosis to ensure that treatment is not delayed, application of numerous adjunct tools in establishing a diagnosis have become relevant, immunohistochemistry is one useful tool. ${ }^{[12]}$

Replacement of the native mucosa by an epithelium resembling the small bowel is defined as intestinal metaplasia (IM). ${ }^{[13,14]}$ Gastric IM is similar histologically to Barrett's esophagus. IM of the gastric mucosa is a relatively frequent precancerous lesion. It is often a source of uncertainty about the appropriate management when included in a gastric biopsy pathology report. ${ }^{[15]}$ Although the risk of gastric cancer is increased in the presence of IM, the overall risk of gastric cancer in a patient with IM is extremely low compared with the risk of adenocarcinoma in a patient with Barrett's esophagus. ${ }^{[16]}$ Cytokeratin are proposed as markers based on their differential patterns of expression in the oesophagus. ${ }^{[17]}$ It is well known that different types of human epithelium have various expressions of CKs which can be used to detect the origin of epithelial cells. CK7 and CK 20 immunohistochemical staining has been used in an attempt to differentiate IM of esophagus versus gastric cardia. Barrett mucosa displays CK20 immunoreactivity of the surface epithelium and superficial glands with absent staining in the deep glands, whereas CK7 strongly highlights both the superficial and deep glands, pattern characteristic of staining of BM. ${ }^{[2,12,18,19]}$ The present study in concurrence with the above demonstrated this CK7 and CK20 pattern in 94\% and $66.7 \%$ cases respectively. On the other hand in our study, CK20 immunoreactivity (94\%) of both superficial and deep glands and surface epithelium was seen in GIM with lack of CK7 staining (88\%) Only $11.8 \%$ cases showed patchy CK7 staining and diffuse CK20 in superficial and deep glands. These findings were similar to patchy CK20 staining of both the superficial and deep glands, with patchy, weak, and variable CK7 labeling in the deep glands with no surface immunoreactivity reported by author. ${ }^{[18,19]}$ On analyzing the staining of intestinal metaplasia of GIM in the present study, CIM showed strong diffuse CK20 staining while there was lack of CK7 expression, IIM on the other hand showed weak patchy CK7 staining and moderate CK20 immunostaining. A positive expression of CK7 in areas of intestinal metaplasia is an evidence of its immature, incomplete or gastrointestinal phenotype, but expression of CK20 is more distinctive for complete, mature/solely small-intestinal phenotype of gastric intestinal metaplasia. ${ }^{[17]}$ MUCs are known to have differential expression patterns in the GIT, the normal gastric mucosa are positive for MUC1, MUC5 AC and MUC6 while it is negative for MUC2, however in case of IM/Barrett's esophagus epithelial cells are positive for MUC2 hence useful in making a diagnosis, staining intensity depends on the number of goblet cells, increased in complete IM and less intense in incomplete IM. ${ }^{[2]}$

Incomplete gastric IM are often the seat for gastric carcinoma, they show staining with cytokeratins, CK 7 and CK20 positivity is seen in both superficial and deep cells of the crypts. ${ }^{[17]}$ Although these results seemed promising, other observers have not been able to reproduce them. ${ }^{[20,21,22]}$ Interpretation of the pattern of staining in BE can be challenging at times, correct assessment relies on proper orientation of the biopsy, identification of both deep glands and surface epithelium as well as presence of IM and absence of dyplasia on hematoxylin-eosin stain. ${ }^{[23]}$ It has been suggested by authors, CK7/CK20 immunostaining pattern is sensitive and specific for $\mathrm{BM}$, and that a semiquantitative CK7/CK20 immunostaining pattern can be used to confirm BE, even when goblet cells are not histologically identified. ${ }^{[12]}$

The cytoskeleton of almost all epithelial cells, cytokeratins, are composed of intermediate filaments encoded by multiple 
genes. These keratin polypeptides are expressed in different cells at different stages of development and differentiation.

${ }^{[24]}$ Carcinoma cells retain an ability to produce the CKs of their progenitor cells, hence are useful biomarkers in the evolution of cancer. ${ }^{[2]}$ A CK7+/CK19+/CK20- pattern was indicative of adenocarcinoma of esophageal origin, in the present study showed CK7+/CK18+/CK19+ in $80 \%$ cases where as gastric adenocarcinoma revealed CK7-/CK20+pattern in $85 \%$ cases and only $40 \%$ showed CK19+ this was similar to expression pattern reported by authors. ${ }^{[25,26]}$ In esophageal SCC, CK7 in $30 \%$, CK20 in $5 \%$ and CK18, CK19 in $95 \%$ and $82 \%$ cases respectively was seen in the present study, while CK18 was present in all the grades of SCC suggesting it to be a useful marker for esophageal SCC, absence of CK20 may be helpful in differential diagnosis. A concordance was seen with findings of Lam KY et.al. ${ }^{[27]}$

The mucin core proteins (MUCs) are glycosylated proteins expressed in tissue specific patterns in the gastrointestinal tract. Their differential expression pattern forms the basis of classifying distinct types of adenocarcinoma as well as carry prognostic significance. ${ }^{[28]}$ Esophageal adenocarcinoma revealed a significantly increased MUC2 expression while MUC1 expression was seen in $85 \%$ cases in the current study. Hence a CK $7+/ C K 20+/ M U C 1+$ pattern is seen in most esophago-gastric junction adenocarcinomas suggested by Flucke et.al. ${ }^{[29]}$ as observed by us, however a difference was seen in the expression of MUC2, $40 \%$ cases were positive in our study while it was absent or faintly detectable by other author. ${ }^{[29]}$

The two markers MUC5AC and MUC2 are useful in elucidating the cell of origin of adenocarcinoma in $\mathrm{BE}$, MUC5AC is specific for gastric type while MUC2 is reflective of intestinal type of adenocarcinoma, none of the two markers are expressed in normal squamous epithelium of the oesophagus. In a study analysis of MUC2 and MUC5AC positivity in distal esophageal adenocarcinomas it showed 17\% MUC2 and 83\% MUC5AC expression pattern. ${ }^{[30]}$ MUC5AC was not analysed in our study. A significant expression of $\mathrm{CK}, \mathrm{MUC1}$ and $\mathrm{MUC2}$ was seen in esophageal adenocarcinoma as compared to SCC, however MUC1 is expressed significantly in poorly differentiated SCC. Kijima H et.al reported 32.1\% MUC1 in SCC as against $50 \%$ in the current study ${ }^{[31]}$ Song ZB et.al. reported high expression of MUC1 is associated with poor prognosis for esophageal cancer patients. ${ }^{[32]} \mathrm{MUC} 2$ is on the other hand useful in gastric adenocarcinoma, it is especially a good marker of mucinous carcinomas (esophageal and gastric).

Expression of all MUC subtypes are aberrant in Barrett's metaplasia. ${ }^{[29,33]}$ Neoplastic progression from Barrett's esophagus to dysplasia and adenocarcinoma is characterized by downregulation of MUC2 and upregulation of MUC1. ${ }^{[33.34]}$ More so, adenocarcinomas of the distal esophagus and gastroesophageal junction if arising in a background of intestinal metaplasia frequently express markers of intestinal differentiation including MUC2. ${ }^{[35]}$ MUC expression in metaplasia both esophageal and gastric is defined by decreased levels of expression of gastric mucin MUC1 and expression of MUC2 intestinal mucin corresponding to type I intestinal metaplasia,while co expression of gastric MUC1 together with MUC2 mucins encompassing type 2 and type 3 intestinal metaplasia, these findings confirm the findings of Celso AR et.al, ${ }^{[36]}$ Silva et.al. ${ }^{[37]}$ MUC1 and MUC2 were expressed in both goblet and non goblet columnar cells in BM while in GIM, MUC1 was expressed in both cells and MUC2 in goblet cells only. These finding were in contradiction to Arul et al. ${ }^{[33]}$ however it was in agreement to others. ${ }^{[36]}$

From the above discussion it is evident that histological similarities between BM and GIM of the cardiac mucosa are a limitation, endoscopic findings are hence crucial in differentiating. Sampling of non representative areas, identification of reactive and dysplastic changes and interobserver variation pose a challenge in arriving at the diagnosis. ${ }^{[9,10]} \mathrm{IHC}$ is a useful adjunct in diagnosing but it has its limitations as progression of disease from preneoplastic to neoplastic lesions is a multistep process, hence no single molecular marker is specific and it is required to develop a panel of markers which in different combinations aid in arriving at the diagnosis.

\section{Conclusions}

Pre Neoplastic and neoplastic lesions of the gastrointestinal tract are varied yet histologically challenging, and can often prove to be a diagnostic dilemma. The significance of establishing a diagnosis lies in the fact that these conditions carry with it a risk of progression to malignancy. Immunoreactivity can be potentially helpful and a useful adjunct however interobserver variability and lack of reproducibility of immunohistochemical results maybe a challenge. Understanding unique immunohistochemical profiles of each entity combined with hematoxylin-eosin examination and endoscopic correlation will greatly assist in the diagnosis and management of these lesions.

\section{References}

1. Memon F, Baloch K, Memon AA. Upper gastrointestinal endoscopic biopsy; morphological spectrum of lesions. Professional Med J 2015;22(12):1574- 1579.

2. Wong $\mathrm{HH}, \mathrm{Chu} \mathrm{P}$. Immunohistochemical features of the gastrointestinal tract tumors. J Gastrointest Oncol 2012;3:262-284. 
3. Ronald M, Werner WF, Renhard et.al. Catalog of human cytokeratin pattern of expressionin normal epithelia tumors and cultured cells. Cell 1982; 31:11-24.

4. Yaron Niv \& Ronnie Fass. The role of mucin in GERD and its complications. Nature Reviews Gastroenterology and Hepatology 2012:9, 55-59

5. Davison JM, Ellis ST, Foxwell TJ, Luketich JD, Gibson MK, Kuan SF, Nason KS. MUC2 Expression is an Adverse Prognostic Factor in Superficial Gastroesophageal Adenocarcinomas. Hum Pathol. 2014;45:540-548.

6. DeMeester SR, Wickramasinghe KS, Lord RV, et al. Cytokeratin and DAS-1 immunostaining reveal similarities among cardiac mucosa, CIM, and Barrett's esophagus. Am J

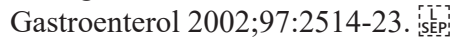

7. Polkowski W, van Lanschot JJ, ten Kate FJ, et al. Intestinal and pancreatic metaplasia at the esophagogastric junction in patients without Barrett's esophagus. Am J Gastroenterol 2000;95:617-25.

8. Spechler SJ. Clinical practice. Barrett's esophagus. N Engl J Med 2002;346:836-42.

9. Amy E. Noffsingerisep. Defining Cancer Risk in Barrett's Esophagus: A Pathologist's Perspective. Gastrointestinal Cancer Research 2008; 2:308-10.

10. Hussain I S, Reshi R, Akhter G, Beigh A.Clinico Histopathological Study Of Upper Gastrointestinal Tract Endoscopic Biopsies. Int J Cur Res Rev 2015; 78-85.

11. Krishnappa R, Horakerappa MS, Karar A, Mangala G. A study on histopathological spectrum of upper gastrointestinal tract endoscopic biopsies. Int J Med Res Health Sci. 2013;2:418-24

12. White N, Gabril M, Ejeckam G, Mathews M, Fardy J, Fady K, Dore J, Yousef GM. Barrett's esophagus and cardiac intestinal metaplasia: Two conditions within the same spectrum. Can J Gastroenterol 2008; 22: 369-75.

13. Bosman FT. World Health Organization.; Inter- national Agency for Research on Cancer. WHO classification of tumors of the digestive system. 4th ed., Lyon: IARC Press,

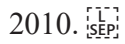

14. Erkan G., Gonul Il, Kandilci U, Dursun A. Eval- uation of apoptosis along with BCL-2 and Ki-67 expression in patients with intestinal metaplasia. Pathol Res Pract 2012;

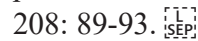

15. Correa P, Piazuelo MB, Wilson KT. Pathology of Gastric Intestinal Metaplasia: Clinical Implications. Am J Gastroenterol. 2010 ; 105: 493-98.

16. Fennerty MB. Gastric intestinal metaplasia on routine endoscopic biopsy. Gastroenterology 2003;125:586-90.

17. Sergii V. Cytokeratins as Molecular Markers in Differential Diagnosis of Gastric Intestinal Metaplasia. J Cytol Tissue Biol 2015; 2: 007.

18. Ormsby AH, Goldblum JR, Rice TW, et al. Cytokeratin subsets can reliably distinguish Barrett's esophagus from intestinal metaplasia of the stomach. Hum Pathol. 1999;30(3):288-294.

19. Ormsby AH, Vaezi MF, Richter JE, et al. Cytokeratin immunoreactivity patterns in the diagnosis of short-segment Barrett's esophagus. Gastroenterology. 2000;119(3):683690.

20. van Baal JW, Bozikas A, Pronk R, et al. Cytokeratin and CDX-2 expression in Barrett's esophagus. Scand J Gastroenterol. 2008;43(2):132-140.

21. Kurtkaya-Yapicier O, Gencosmanoglu R, Avsar E, Bakirci N, Tozun N, Sav A. The utility of cytokeratins 7 and 20 (CK7/20) immunohistochemistry in the distinction of short-segment Barrett esophagus from gastric intestinal metaplasia: is it reliable? BMC Clin Pathol. 2003;3(1):5.

22. Gulmann C, Shaqaqi OA, Grace A, et al. Cytokeratin 7/20 and MUC1, 2, 5AC, and 6 expression patterns in Barrett's esophagus and intestinal metaplasia of the stomach: intestinal metaplasia of the cardia is related to Barrett's esophagus. Appl Immunohistochem Mol Morphol. 2004;12(2):142147.

23. Voltaggio L, Montgomery EA, Lam- Himlin D. A Clinical and Histopathologic Focus on Barrett Esophagus and BarrettRelated Dysplasia. Arch Pathol Lab Med. 2011;135:1249 1260 .

24. Singh A, Kapur S, Chattopadhyay I, Purkayastha J, Sharma J, Mishra A, Hewitt SM, Saxena S. Cytokeratin immunoexpression in esophageal squamous cell carcinoma of high-risk population in Northeast India. Appl Immunohistochem Mol Morphol. 2009 ; 17:419-24.

25. Philippe T, Giovanna BS, Saurin JC, Lombard- Bohas C, Boulez J, Berger F.et.al. Cytokeratin expression in adenocarcinoma of esophagogastric junction: A comparative study of adenocarcinoma of the Distal esophagus and of the stomach.Am J of Surg Pathol. 2002;26:1213-21.

26. Ectors N, Driessen A, Hertog G, Lerut T, Geboes K. Is Adenocarcinoma of the Esophagogastric Junction or Cardia Different From Barrett Adenocarcinoma? Arch Pathol Lab Med. 2005;128:183-85.

27. Lam KY, Loke SL, Laifer J.Cytokeratin pattern in non neoplastic esophageal epithelium and squamous cell carcinoma. Virchows Arsh 1995;426:345-349.

28. Davison JM, Ellis ST, Foxwell TJ, Luketich JD, Gibson MK, , Kuan S et.al. MUC2 Expression is an Adverse Prognostic Factor in Superficial Gastroesophageal Adenocarcinomas. Hum Pathol. 2014; 45: 540-48.

29. Flucke U, Steinborn E, Dries V, et al. Immunoreactivity of cytokeratins (CK7, CK20) and mucin peptide core antigens (MUC1, MUC2, MUC5AC) in adenocarcinomas, normal and metaplastic tissues of the distal oesophagus, oesophagogastric junction and proximal stomach. Histopathology. 2003; 43:127-34.

30. Di Maio MA, Kwok S, Montgomery KD, Lowe AW, Pai RK. Immunohistochemical Panel for Distinguishing Esophageal 
Adenocarcinoma from Squamous Cell Carcinoma: A Combination of p63, Cytokeratin 5/6, MUC5AC, and AGR2 Allows Optimal Subtyping. Hum Pathol. 2012; 43: 17991807.

31. Kijima H, Chino O, Yamazaki H. Immunohistochemical MUC1 (DF3 antigen) expression of human esophageal squamous cell carcinoma. Anticancer. Res. 2001;21:1285-89.

32. Song ZB, Gao SS, Yi XN. Expression of MUC1 in esophageal squamous cell carcinoma and its relationship with poor prognosis of patients from Linzhou city, a high incidence of northern China. World J Gastroentrology.2003; 9:404-07.

33. Arul GS, Moorghen M, Myerscough N, Alderson DA, Spicer RD, Corfield AP. Mucin gene expression in Barrett's oesophagus: an in situ hybridisation and immunohistochemical study. Gut. 2000; 47:753-761.

34. Chinyama CN, Marshall RE, Owen WJ, Mason RC, Kothari D, Wilkinson ML, Sanderson JD. Expression of MUC1 and MUC2 mucin gene products in Barrett's metaplasia, dysplasia and adenocarcinoma: an immunopathological study with clinical correlation. Histopathology. 1999; 35:517-524.

35. Demicco EG, Farris AB 3rd, Baba Y, Agbor-Etang B, Bergethon K, Mandal R, Daives D, Fukuoka J, Shimizu M, Dias-Santagata D, Ogino S, Iafrate AJ, Gaissert HA, MinoKenudson M. The dichotomy in carcinogenesis of the distal esophagus and esophagogastric junction: intestinal-type vs cardiac-type mucosa-associated adenocarcinoma. Mod Pathol. 2011; 24:1177-1190.

36. Celso AR, Leonor D, Correa P. Intestinal metaplasia of stomach displays distinct patterns of mucin (MUC1, MUC2, MUC5AC, MUC6) expression. Cancer Res.1991;59: 1003-07.

37. Silva E, Teixeira A, David L. Mucins as key molecules for the classifications of intestinal metaplasia of stomach. Virchows Arch. 2002;440:311-17.

*Corresponding author:

Dr Zeeba Shamim Jairajpuri, Associate Professor, Department of Pathology Hamdard Institute of Medical Sciences and Research Jamia Hamdard, New Delhi 110062, India

Email: jairajpurizs@gmail.com

Financial or other Competing Interests: None. 